\title{
CARBON CHEMISTRY IN THE ENVELOPE OF VY CANIS MAJORIS: IMPLICATIONS FOR OXYGEN-RICH EVOLVED STARS
}

\author{
L. M. Ziurys ${ }^{1,2,3}$, E. D. Tenenbaum ${ }^{1,2}$, R. L. Pulliam ${ }^{1,2}$, N. J. Woolf ${ }^{1}$, and S. N. Milam ${ }^{4}$ \\ ${ }^{1}$ Department of Astronomy and Steward Observatory, University of Arizona, 933 N. Cherry Ave., Tucson, AZ 85721, USA; lziurys@ as.arizona.edu, \\ emilyt@as.arizona.edu, rpulliam@email.arizona.edu, nwoolf@as.arizona.edu \\ ${ }^{2}$ Department of Chemistry, University of Arizona, Tucson, AZ 85721, USA \\ ${ }^{3}$ Arizona Radio Observatory, University of Arizona, Tucson, AZ 85721, USA \\ ${ }^{4}$ SETI Institute, NASA Ames Research Center, M/S 245-6, Moffet Field, CA 94035, USA; Stefanie.N.Milam@nasa.gov \\ Received 2008 October 12; accepted 2009 January 13; published 2009 April 8
}

\begin{abstract}
Observations of the carbon-bearing molecules $\mathrm{CO}, \mathrm{HCN}, \mathrm{CS}, \mathrm{HNC}, \mathrm{CN}$, and $\mathrm{HCO}^{+}$have been conducted toward the circumstellar envelope of the oxygen-rich red supergiant star, VY Canis Majoris (VY CMa), using the Arizona Radio Observatory (ARO). CO and HCN were also observed toward the O-rich shells of NML Cyg, TX Cam, IK $\mathrm{Tau}$, and W Hya. Rotational transitions of these species at $1 \mathrm{~mm}, 0.8 \mathrm{~mm}$, and $0.4 \mathrm{~mm}$ were measured with the ARO Submillimeter Telescope, including the $J=6 \rightarrow 5$ line of CO at $691 \mathrm{GHz}$ toward TX Cam and W Hya. The ARO $12 \mathrm{~m}$ was used for $2 \mathrm{~mm}$ and $3 \mathrm{~mm}$ observations. Four transitions were observed for $\mathrm{HCO}^{+}$in $\mathrm{VY} \mathrm{CMa}$, the first definitive identification of this ion in a circumstellar envelope. Molecular line profiles from VY CMa are complex, indicating three separate outflows: a roughly spherical flow and separate red- and blueshifted winds, as suggested by earlier observations. Spectra from the other sources appear to trace a single outflow component. The line data were modeled with a radiative transfer code to establish molecular abundances relative to $\mathrm{H}_{2}$ and source distributions. Abundances for CO derived for these objects vary over an order of magnitude, $f \sim 0.4-5 \times 10^{-4}$, with the lower values corresponding to the supergiants. For HCN, a similar range in abundance is found $(f \sim 0.9-9$ $\times 10^{-6}$ ), with no obvious dependence on the mass-loss rate. In VY CMa, $\mathrm{HCO}^{+}$is present in all three outflows with $f \sim 0.4-1.6 \times 10^{-8}$ and a spatial extent similar to that of CO. HNC is found only in the red- and blueshifted components with $[\mathrm{HCN}] /[\mathrm{HNC}] \sim 150-190$, while $[\mathrm{CN}] /[\mathrm{HCN}] \sim 0.01$ in the spherical flow. All three velocity components are traced in CS, which has a confined spatial distribution and $f \sim 2-6 \times 10^{-7}$. These observations suggest that carbon-bearing molecules in O-rich shells are produced by a combination of photospheric shocks and photochemistry. Shocks may play a more prominent role in the supergiants because of their macroturbulent velocities.
\end{abstract}

Key words: astrochemistry - circumstellar matter - radio lines: stars - stars: AGB and post-AGB - supergiants

\section{INTRODUCTION}

Studies of circumstellar envelopes of evolved stars over the past few decades have suggested that the $\mathrm{C} / \mathrm{O}$ ratio governs the chemistry of these objects (e.g., Tsuji 1973; Scalo and Slavsky 1980; Mamon et al. 1987). In stars where $\mathrm{C}>\mathrm{O}$, the oxygen is thought to be almost entirely in the form of carbon monoxide with little remaining for other molecules. In the case where $\mathrm{C}<\mathrm{O}$, the majority of the carbon is proposed to be contained in $\mathrm{CO}$ such that abundances of other $\mathrm{C}$-bearing species are negligible. However, more recent observations suggest that $\mathrm{CO}$ may not be the deciding species after all. $\mathrm{H}_{2} \mathrm{O}, \mathrm{H}_{2} \mathrm{CO}$, and even $\mathrm{C}_{3} \mathrm{O}$ have now been found in the shell of the extreme C-rich asymptotic giant branch (AGB) star IRC+10216 (e.g., Melnick et al. 2001; Ford et al. 2004; Tenenbaum et al. 2006). Furthermore, HCN appears to be present in at least $50 \%$ of all O-rich circumstellar shells, including red giants, AGB stars, and supergiants (e.g., Nercessian et al. 1989; Olofsson et al. 1998). In certain cases, such as the O-rich AGB stars TX Cam and IK Tau, CS and CN have also been observed (Olofsson et al. 1991, 1998; Bachiller et al. 1997), adding further complexity to the issue. There has also been some speculation that the HCN abundance depends on the mass-loss rate (Olofsson et al. 1998). More recent work suggests that "parent" molecules such as HCN, $\mathrm{CS}$, and CO are commonly present in all AGB circumstellar shells, independent of their evolutionary stage, first predicted theoretically (Cherchneff 2006) and then tested observationally (Decin et al. 2008).
Models of circumstellar chemistry can reproduce many of these unexpected observed abundances by invoking ionmolecule and radical-radical processes in the outer envelope. Agúndez \& Cernicharo (2006), for example, account for many of the O-containing molecules found in IRC+10216 using reactions based on atomic oxygen, produced by the photodissociation of CO in the outer shell. Both Willacy \& Millar (1997) and Nejad \& Millar (1988) explain the presence of HCN in O-rich shells with chemical pathways initiated by the photodestruction products of $\mathrm{CH}_{4}: \mathrm{CH}, \mathrm{CH}_{2}$, and $\mathrm{CH}_{3}$. A large abundance of $\mathrm{CH}_{4}$ is needed in this case. The $\mathrm{HCN}$ observations of Olofsson et al. (1998) suggest such a photochemical origin. More recent models, on the other hand, employ photospheric shocks as a means of creating carbon-bearing molecules in O-rich environments (Duari et al. 1999; Cherchneff 2006). The high temperatures generated in the postshocked gas enable neutral-neutral reactions to occur that result in high concentrations of radicals such as $\mathrm{CN}$, that subsequently lead to $\mathrm{HCN}$ and CS. High methane concentrations are not needed in this scenario. These calculations have successfully reproduced some of the observed abundances for such species as $\mathrm{CO}_{2}$ and $\mathrm{HCN}$ (Duari et al. 1999; Bieging et al. 2000).

One of the oxygen-rich stars that has recently been found to exhibit a complex chemistry is the supergiant VY Canis Majoris, or VY CMa (Ziurys et al. 2007). Unusual molecules such as $\mathrm{NaCl}$ and $\mathrm{PO}$ have been detected in the circumstellar shell of this star (Milam et al. 2009; Tenenbaum et al. 2007). A surprising 
Table 1

Properties of Observed O-Rich Envelopes

\begin{tabular}{lcccccl}
\hline \hline Source & $\alpha(\mathrm{B} 1950.0)$ & $\delta(\mathrm{B} 1950.0)$ & $R_{*}(\mathrm{~cm})$ & $T_{*}(\mathrm{~K})$ & Type & \multicolumn{1}{c}{ References } \\
\hline VY CMa & $07^{\mathrm{h}} 20^{\mathrm{m}} 54^{\mathrm{s}} .7$ & $-25^{\circ} 40^{\prime} 12^{\prime \prime}$ & $2.0 \times 10^{14}$ & 2800 & Red supergiant & Zubko et al. (2004) \\
NML Cyg & $20^{\mathrm{h}} 44^{\mathrm{m}} 33^{\mathrm{s}} .8$ & $39^{\circ} 55^{\prime} 57^{\prime \prime}$ & $2.6 \times 10^{14}$ & 2500 & Red supergiant & Monnier et al. (1997) \\
TX Cam & $04^{\mathrm{h}} 56^{\mathrm{m}} 40^{\mathrm{s}} 6$ & $56^{\circ} 06^{\prime} 28^{\prime \prime}$ & $2.0 \times 10^{13}$ & 2600 & AGB & Olofsson et al. (1991) \\
IK Tau & $03^{\mathrm{h}} 50^{\mathrm{m}} 43^{\mathrm{s}} .6$ & $11^{\circ} 15^{\prime} 32^{\prime \prime}$ & $2.1 \times 10^{13}$ & 2100 & AGB & Duari et al. (1999) \\
W Hya & $13^{\mathrm{h}} 46^{\mathrm{m}} 12^{\mathrm{s}} .2$ & $-28^{\circ} 07^{\prime} 07^{\prime \prime}$ & $2.7 \times 10^{13}$ & 2500 & AGB & Justtanont et al. (2005) \\
\hline
\end{tabular}

number of carbon-bearing molecules have been observed as well, including $\mathrm{HNC}$ and $\mathrm{HCO}^{+}$, as mentioned briefly in Ziurys et al. (2007). In this paper we present the observations of carbon-bearing molecules in VY CMa, a detailed analysis of these data to establish abundances, and a comparison of these results with current models. We also present comparative observations of $\mathrm{CO}$ and $\mathrm{HCN}$ in other O-rich envelopes. In every case, multiple transitions were measured such that accurate abundances could be established. Here we describe these results and their implications for the chemistry in O-rich circumstellar shells.

\section{OBSERVATIONS}

The data were collected during the period 2005 December to 2008 March using the Arizona Radio Observatory's (ARO) $12 \mathrm{~m}$ dish on Kitt Peak and $10 \mathrm{~m}$ Submillimeter Telescope (SMT) on Mt. Graham. Source coordinates are given in Table 1, and observing frequencies, beam efficiencies, and beam sizes are listed in Table 2. Observations were conducted in beamswitching mode with a $\pm 2^{\prime}$ beam throw, and local oscillator shifts were done to check for image contamination. Pointing and focus were monitored regularly on planets and quasars.

The 2 and $3 \mathrm{~mm}$ measurements were carried out at the $12 \mathrm{~m}$ telescope using single-sideband dual-polarization SIS mixers with typical image rejection $\geqslant 17 \mathrm{~dB}$. The backends consisted of 1 and $2 \mathrm{MHz}$ resolution filterbanks operated in parallel $(2 \times$ 256 channels) mode, and a $782 \mathrm{kHz}$ resolution autocorrelator. The intensity scale at the $12 \mathrm{~m}$ is given as $T_{R}^{*}$, which is the chopper-wheel antenna temperature corrected for forward spillover losses. Radiation temperature is then defined as $T_{R}=$ $T_{R}^{*} / \eta_{\mathrm{c}}$, where $\eta_{\mathrm{c}}$ is the corrected beam efficiency.

Data in the $1,0.8$, and $0.4 \mathrm{~mm}$ windows were collected at the SMT using a suite of three receivers. The temperature scale at the SMT is given in $T_{A}^{*}$, the chopper-wheel antenna temperature. Here the radiation temperature is defined as $T_{R}=T_{A}^{*} / \eta_{\mathrm{b}}$, where $\eta_{\mathrm{b}}$ is the main-beam efficiency. The $1 \mathrm{~mm}$ observations employed a dual-polarization ALMA Band 6 receiver system featuring sideband-separating mixers with typical image rejection of $15-20 \mathrm{~dB}$. Measurements at $0.8 \mathrm{~mm}(340-360 \mathrm{GHz})$ were carried out using a double-sideband dual-polarization receiver. A new receiver operating from 620 to $710 \mathrm{GHz}$ was used to obtain spectra of the CO: $J=6 \rightarrow 5$ transition at $691 \mathrm{GHz}$. This double-sideband system, which at the time of these observations was single polarization, features ALMA Band 9 mixers. Typical system temperatures at $691 \mathrm{GHz}$ under good observing conditions were $T_{\text {sys }} \sim 1600 \mathrm{~K}$, DSB. The temperature scale for the double sideband receivers was established assuming equal gain in either sideband. Filter banks with 2048 channels at $1 \mathrm{MHz}$ resolution were the backends used for all SMT measurements, typically operated in parallel mode $(2 \times 1024$ channels $)$.

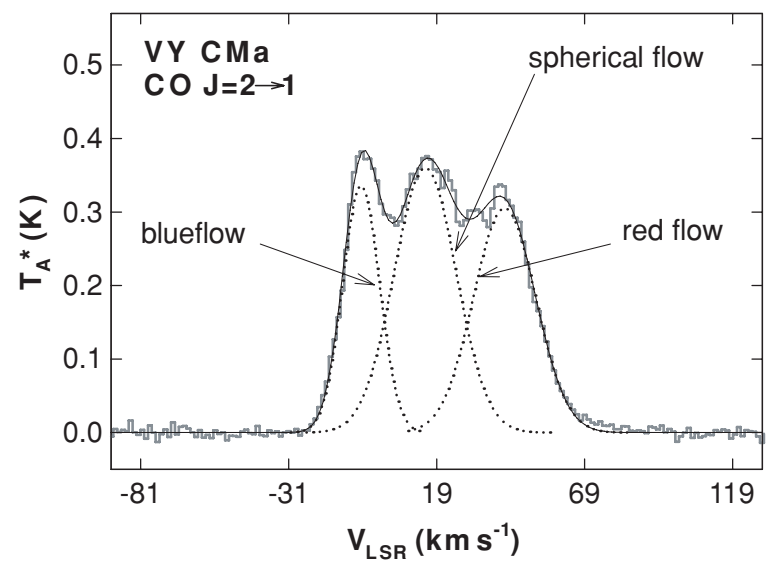

Figure 1. Spectrum of the $J=2 \rightarrow 1$ transition of $\mathrm{CO}$ from Ziurys et al. (2007), observed toward VY CMa at $230 \mathrm{GHz}$, using the ARO SMT with 1 $\mathrm{MHz}$ spectral resolution. This spectrum consists of three velocity components, as indicated in the figure by dotted curves: a spherical outflow at the systemic velocity of the star, a blueshifted wind near $V_{\mathrm{LSR}}=-5 \mathrm{~km} \mathrm{~s}^{-1}$, and a redshifted flow with $V_{\mathrm{LSR}} \sim 45 \mathrm{~km} \mathrm{~s}^{-1}$. The line profile has been modeled with these three components and the overall fit is shown overlying the spectrum as a solid line.

\section{RESULTS}

Six carbon-bearing molecules were observed in VY CMa: $\mathrm{CO}, \mathrm{CS}, \mathrm{HCN}, \mathrm{HNC}, \mathrm{CN}$, and $\mathrm{HCO}^{+}$. While $\mathrm{CO}, \mathrm{HCN}$, and $\mathrm{CN}$ had been identified previously in this object (e.g., Nercessian et al. 1989; Bachiller et al. 1997; Kemper et al. 2003), HNC, $\mathrm{CS}$, and $\mathrm{HCO}^{+}$are new detections for VY CMa, as described in Ziurys et al. (2007). $\mathrm{HCO}^{+}$had not been observed previously in an O-rich giant, supergiant, or AGB envelope. Several transitions were observed for each molecule, as shown in Table 2, supporting these identifications. Apparent line parameters for each observed feature are given in Table 2, including LSR velocity, line intensity (in $T_{A}^{*}$ or $\left.T_{R}^{*}\right)$, linewidth at half-power $\left(\Delta \mathrm{V}_{1 / 2}\right)$, and integrated line intensity $\int T_{R}^{*} d v$ or $\int T_{A}^{*} d v$. As this table illustrates, the line temperatures are all $<1 \mathrm{~K}$ and the range of velocities is $V_{\mathrm{LSR}} \sim 19-22 \mathrm{~km} \mathrm{~s}^{-1}$-characteristic of VY CMa (e.g., Kemper et al. 2003). The linewidths are also broadtypically $55-70 \mathrm{~km} \mathrm{~s}^{-1}$ - but they are not uniformly the same. This variation arises from the fact that VY CMa has at least three separate outflows that are not traced by every molecule in all transitions (see Ziurys et al. 2007). The presence or absence of the distinct velocity components produces different line profiles.

The three outflow components are visible in the spectrum of the $J=2 \rightarrow 1$ transition of CO, shown in Figure 1. A Gaussian fit to this profile is shown overlaying the spectrum as a solid line, as well as its decomposition into three outflows, indicated on the figure as dashed curves. The spectrum appears to consist of a collimated, blueshifted wind near $V_{\mathrm{LSR}} \approx-5 \mathrm{~km} \mathrm{~s}^{-1}$, a more diffuse redshifted flow with $V_{\mathrm{LSR}} \sim 45 \mathrm{~km} \mathrm{~s}^{-1}$, and a roughly spherical flow, centered at the systemic velocity of the star of 
Table 2

Line Parameters of Observed Transitions

\begin{tabular}{|c|c|c|c|c|c|c|c|c|c|}
\hline Source & Molecule & Transition & Frequency (MHz) & $\eta_{\mathrm{b}}$ or $\eta_{\mathrm{c}}$ & $\theta_{\mathrm{b}}\left({ }^{\prime \prime}\right)$ & $V_{\text {LSR }}\left(\mathrm{km} \mathrm{s}^{-1}\right)$ & $T_{A}^{*}$ or $T_{R}^{* \text { a }}(\mathrm{K})$ & $\Delta \mathrm{V}_{1 / 2}\left(\mathrm{~km} \mathrm{~s}^{-1}\right)$ & $\bar{c} \int T_{\mathrm{A}}{ }^{*} \mathrm{dV}$ or $\int \mathrm{T}_{\mathrm{R}}{ }^{*} \mathrm{dV}^{\mathrm{a}}\left(\mathrm{K} \mathrm{km} \mathrm{s}^{-1}\right)$ \\
\hline \multirow[t]{17}{*}{ VY CMa } & \multirow[t]{3}{*}{$\mathrm{CO}^{\mathrm{b}}$} & $J=1 \rightarrow 0$ & 115271.2 & 0.85 & 55 & $24.6(5.2)$ & $\sim 0.043$ & $73.5(5.2)$ & $2.93(0.34)$ \\
\hline & & $J=2 \rightarrow 1$ & 230538.0 & 0.78 & 33 & $19.8(3.9)$ & $\sim 0.35$ & $67.0(3.9)$ & $22.93(0.60)$ \\
\hline & & $J=3 \rightarrow 2$ & 345796.0 & 0.70 & 22 & $20.6(3.5)$ & $\sim 0.7$ & $67(3)$ & $46.9(3.0)$ \\
\hline & \multirow[t]{2}{*}{$\mathrm{HCN}^{\mathrm{b}}$} & $J=1 \rightarrow 0$ & 88631.6 & 0.90 & 71 & $19.0(6.8)$ & $0.047(0.007)$ & $68.5(6.8)$ & $2.98(0.29)$ \\
\hline & & $J=3 \rightarrow 2$ & 265886.4 & 0.78 & 28 & $20.1(3.6)$ & $0.400(0.010)$ & $55.4(3.6)$ & $23.04(0.50)$ \\
\hline & \multirow[t]{2}{*}{$C S^{b}$} & $J=3 \rightarrow 2$ & 146969.0 & 0.75 & 43 & $19(4)$ & $0.003(0.002)$ & $74(12)$ & $0.27(0.20)$ \\
\hline & & $J=5 \rightarrow 4$ & 244935.6 & 0.78 & 31 & $22.7(2.4)$ & $0.014(0.003)$ & $70.0(4.8)$ & $0.98(0.26)$ \\
\hline & \multirow[t]{2}{*}{$\mathrm{HNC}^{\mathrm{c}}$} & $J=1 \rightarrow 0$ & 90663.6 & 0.88 & 69 & $\sim 19$ & $0.0015(0.0010)$ & $\sim 75$ & $\ldots$ \\
\hline & & $J=3 \rightarrow 2$ & 271981.1 & 0.78 & 28 & $21.7(2.2)$ & $0.018(0.005)$ & $64(2.2)$ & $0.85(0.25)$ \\
\hline & \multirow[t]{4}{*}{$\mathrm{CN}^{\mathrm{d}}$} & $N=1 \rightarrow 0$ & & & & & & & \\
\hline & & $\begin{array}{c}J=3 / 2 \rightarrow 1 / 2^{\mathrm{e}} \\
N=2 \rightarrow 1\end{array}$ & 113490.9 & 0.85 & 55 & $19.0(7.8)$ & $0.005(0.004)$ & $62.3(10.4)$ & $0.34(0.10)$ \\
\hline & & $J=3 / 2 \rightarrow 1 / 2^{\mathrm{f}}$ & 226658.9 & 0.78 & 33 & $21.6(2.6)$ & $0.009(0.004)$ & $73.1(5.2)$ & $0.64(0.41)$ \\
\hline & & $J=5 / 2 \rightarrow 3 / 2^{\mathrm{g}}$ & 226876.4 & 0.78 & 33 & $20.0(2.6)$ & $0.013(0.004)$ & $53.0(5.2)$ & $0.68(0.24)$ \\
\hline & \multirow[t]{4}{*}{$\mathrm{HCO}^{+b}$} & $J=1 \rightarrow 0$ & 89188.5 & 0.89 & 70 & $19.0(6.7)$ & $0.010(0.002)$ & $67.4(6.7)$ & $0.54(0.18)$ \\
\hline & & $J=2 \rightarrow 1$ & 178375.1 & 0.68 & 35 & $20.9(3.3)$ & $0.020(0.006)$ & $73.0(6.7)$ & $0.85(0.11)$ \\
\hline & & $J=3 \rightarrow 2^{\mathrm{h}}$ & 267557.6 & 0.78 & 28 & $\sim 19$ & $\sim 0.025$ & $\sim 70$ & $\ldots$ \\
\hline & & $J=4 \rightarrow 3^{\mathrm{i}}$ & 356734.2 & 0.70 & 21 & $\sim 19$ & $\sim 0.03$ & $\sim 70$ & $\ldots$ \\
\hline \multirow[t]{5}{*}{ NML Cyg } & \multirow[t]{3}{*}{$\mathrm{CO}$} & $J=1 \rightarrow 0^{\mathrm{j}}$ & 115271.2 & 0.85 & 55 & $\sim-5$ & $\sim 0.2$ & $\sim 50$ & $\sim 8$ (1) \\
\hline & & $J=2 \rightarrow 1$ & 230538.0 & 0.78 & 33 & $0.5(2.6)$ & $0.706(0.015)$ & $48.1(1.3)$ & $32.1(0.4)$ \\
\hline & & $J=6 \rightarrow 5$ & 691473.1 & 0.33 & 11 & $4(8)$ & $0.9(0.3)$ & $44(9)$ & $35(15)$ \\
\hline & \multirow[t]{2}{*}{$\mathrm{HCN}$} & $J=1 \rightarrow 0$ & 88631.6 & 0.90 & 71 & $-1(7)$ & $0.024(0.008)$ & $54.1(6.8)$ & $1.3(0.3)$ \\
\hline & & $J=3 \rightarrow 2$ & 265886.4 & 0.78 & 28 & $1.9(4.4)$ & $0.23(0.08)$ & $43.3(4.4)$ & $10.3(2.6)$ \\
\hline \multirow[t]{5}{*}{ W Hya } & \multirow[t]{3}{*}{$\mathrm{CO}$} & $J=1 \rightarrow 0$ & 115271.2 & 0.85 & 55 & 40.7 (2.6) & $0.045(0.008)$ & $10.0(2.6)$ & $0.53(0.15)$ \\
\hline & & $J=2 \rightarrow 1$ & 230538.0 & 0.78 & 33 & $40.8(1.3)$ & $0.33(0.02)$ & $14.2(2.6)$ & $4.44(0.17)$ \\
\hline & & $J=6 \rightarrow 5$ & 691473.1 & 0.33 & 11 & $41.4(2.6)$ & $1.26(0.42)$ & $13(3)$ & $15(7)$ \\
\hline & \multirow[t]{2}{*}{$\mathrm{HCN}$} & $J=1 \rightarrow 0$ & 88631.6 & 0.90 & 71 & $42.2(3.4)$ & $0.025(0.006)$ & $13.9(3.4)$ & $0.36(0.14)$ \\
\hline & & $J=3 \rightarrow 2$ & 265886.4 & 0.78 & 28 & 40.7 (1.1) & $0.376(0.006)$ & $8.0(2.2)$ & $3.04(0.10)$ \\
\hline \multirow[t]{5}{*}{ TX Cam } & \multirow[t]{3}{*}{$\mathrm{CO}$} & $J=1 \rightarrow 0$ & 115271.2 & 0.85 & 55 & $10.8(2.6)$ & $0.30(0.04)$ & $28.4(2.6)$ & $8.23(0.99)$ \\
\hline & & $J=2 \rightarrow 1$ & 230538.0 & 0.78 & 33 & $11.1(2.6)$ & $0.76(0.01)$ & $28.3(2.6)$ & $20.89(0.50)$ \\
\hline & & $J=6 \rightarrow 5$ & 691473.1 & 0.33 & 11 & $12.4(1.7)$ & $0.8(0.2)$ & $28.6(6.1)$ & $25.0(7.6)$ \\
\hline & \multirow[t]{2}{*}{$\mathrm{HCN}$} & $J=1 \rightarrow 0$ & 88631.6 & 0.90 & 71 & $13.3(6.8)$ & $0.088(0.006)$ & $27.9(6.8)$ & $2.51(0.35)$ \\
\hline & & $J=3 \rightarrow 2$ & 265886.4 & 0.78 & 28 & $12.7(2.2)$ & $0.30(0.01)$ & $20.2(2.2)$ & $6.35(0.46)$ \\
\hline \multirow[t]{4}{*}{ IK Tau } & \multirow[t]{2}{*}{$\mathrm{CO}$} & $J=1 \rightarrow 0$ & 115271.2 & 0.85 & 55 & $34.9(2.6)$ & $0.43(0.01)$ & $31.6(2.6)$ & $14.45(0.59)$ \\
\hline & & $J=2 \rightarrow 1$ & 230538.0 & 0.78 & 33 & $34.5(1.3)$ & $1.22(0.01)$ & $32.7(1.3)$ & $36.43(0.24)$ \\
\hline & \multirow[t]{2}{*}{$\mathrm{HCN}$} & $J=1 \rightarrow 0$ & 88631.6 & 0.90 & 71 & $37.4(3.4)$ & $0.047(0.007)$ & $36.9(3.4)$ & $1.79(0.40)$ \\
\hline & & $J=3 \rightarrow 2$ & 265886.4 & 0.78 & 28 & $35.3(2.2)$ & $0.221(0.016)$ & $27.3(2.2)$ & $5.56(0.56)$ \\
\hline
\end{tabular}

Notes.

a $T_{A}^{*}$ applies to SMT data and $T_{R}^{*}$ to $12 \mathrm{~m}$ data.

${ }^{\mathrm{b}}$ Line parameters measured for the combined redshifted, blueshifted, and central flow emission (see text).

${ }^{c}$ Line parameters measured for the combined red- and blueshifted flow emission (see text).

${ }^{\mathrm{d}}$ Line parameters measured for the designated fine structure components; specific flows could not be discerned due to hyperfine splitting.

e Blend of hyperfine components: $F=3 / 2 \rightarrow 1 / 2,5 / 2 \rightarrow 3 / 2,1 / 2 \rightarrow 1 / 2,3 / 2 \rightarrow 3 / 2,1 / 2 \rightarrow 3 / 2$.

${ }^{\mathrm{f}}$ Blend of hyperfine components: $F=1 / 2 \rightarrow 3 / 2,3 / 2 \rightarrow 3 / 2,5 / 2 \rightarrow 3 / 2,1 / 2 \rightarrow 1 / 2,3 / 2 \rightarrow 1 / 2$.

g Blend of hyperfine components: $F=5 / 2 \rightarrow 3 / 2,7 / 2 \rightarrow 5 / 2,3 / 2 \rightarrow 1 / 2,3 / 2 \rightarrow 3 / 2,5 / 2 \rightarrow 5 / 2,3 / 2 \rightarrow 5 / 2$.

h Contaminated by the $J_{\mathrm{Ka}, \mathrm{Kc}}=13_{3,11} \rightarrow 13_{2,12}$ transition of $\mathrm{SO}_{2}$ at $267537.5 \mathrm{MHz}$.

${ }^{\mathrm{i}}$ Contaminated by the $J_{\mathrm{Ka}, \mathrm{Kc}}=10_{4,6} \rightarrow 10_{3,7}$ transition of $\mathrm{SO}_{2}$ at $356755.2 \mathrm{MHz}$.

j Highly contaminated by Galactic CO.

$19 \mathrm{~km} \mathrm{~s}^{-1}$. This spherical flow, exclusively present in line profiles of $\mathrm{SiO}, \mathrm{PN}$, and $\mathrm{NaCl}$ (Ziurys et al. 2007), is characterized by $\Delta \mathrm{V}_{1 / 2} \sim 30-40 \mathrm{~km} \mathrm{~s}^{-1}$ — significantly more narrow than most of the apparent linewidths measured here.

These components are visible in the spectra of the other carbon-bearing species, displayed in Figures 2-4. In Figure 2, spectra of $\mathrm{HCN}(J=1 \rightarrow 0$ and $3 \rightarrow 2)$, HNC $(J=3 \rightarrow 2)$, and $\mathrm{CN}(N=2 \rightarrow 1)$ are presented. In the case of $\mathrm{CN}$, two fine structure components are shown $(J=5 / 2 \rightarrow 3 / 2$ and $3 / 2 \rightarrow$ $1 / 2$ ), each of which consists of multiple hyperfine transitions whose positions and relative intensities are indicated underneath the spectrum (also see Milam et al. 2009) The contrast between the $J=3 \rightarrow 2$ lines of $\mathrm{HCN}$ and $\mathrm{HNC}$ is striking. The HNC profile consists exclusively of the blue- and redshifted outflows (see Figure 1). The HCN line appears Gaussian in shape, but is too broad-greater than $55 \mathrm{~km} \mathrm{~s}^{-1}$ - to only trace the spherical flow. The shape of the HCN line profile results from the presence of all three components. Emission from the spherical flow "fills in" the profile between the red- and blueshifted wings. When the blue- and redshifted flows are prominent in the spectra, a more "boxy" profile results, as seen in the $J=1 \rightarrow 0$ line of $\mathrm{HCN}$ (top panel: Figure 2) and in the $J=2 \rightarrow 1$ transition of CO (Figure 1). As these components diminish in intensity, the profile becomes more Gaussian in appearance, as illustrated by the $J=$ $3 \rightarrow 2$ transition of $\mathrm{HCN}$. The linewidths thus are an important quantity in evaluating the origin of any particular molecule. In the case of $\mathrm{CN}$, however, the presence of multiple hyperfine components makes evaluation of the line profiles uncertain. The 


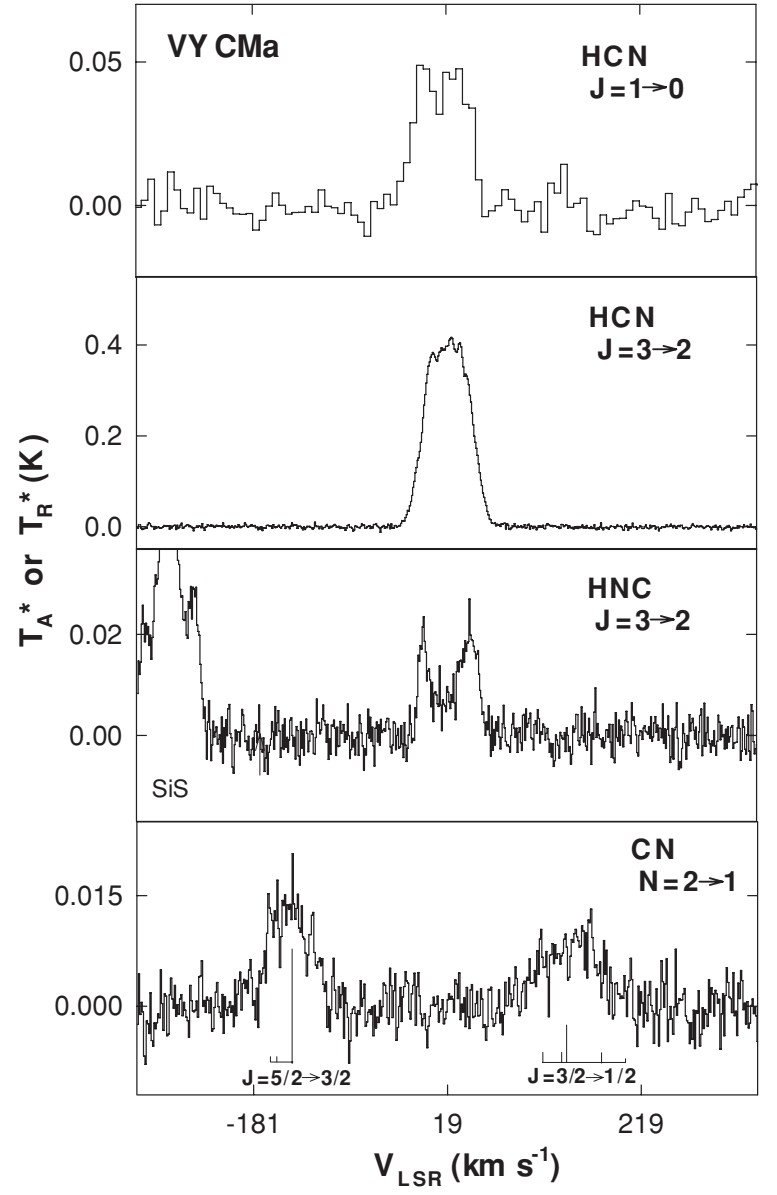

Figure 2. Spectra of the $J=3 \rightarrow 2$ and $1 \rightarrow 0$ transitions of $\mathrm{HCN}$, the $J=3 \rightarrow 2$ line of HNC, and two spin-rotation components, labeled by $J$, of the $N=2 \rightarrow 1$ transition of $\mathrm{CN}$, observed toward the circumstellar shell of the supergiant star VY CMa. All $1 \mathrm{~mm}$ data were measured with the ARO SMT in units of $T_{A}^{*}$, except the $J=1 \rightarrow 0$ line of $\mathrm{HCN}$ at $88 \mathrm{GHz}$, which was observed with the $12 \mathrm{~m}$ where the temperature scale is $T_{R}^{*}$. Spectral resolution is $1 \mathrm{MHz}$ for the SMT data and $2 \mathrm{MHz}$ for the $12 \mathrm{~m}$ spectrum. The positions and relative intensities of the hyperfine components of $\mathrm{CN}$ are marked underneath the spectrum, which has a center frequency of $226,754 \mathrm{MHz}$ for $V_{\mathrm{LSR}}=19 \mathrm{~km} \mathrm{~s}^{-1}$. The line shapes of $\mathrm{HCN}$ and $\mathrm{HNC}$ vary significantly; the latter molecule does not appear to be present in the spherical outflow from the star, but only in redand blueshifted winds. HCN traces all three velocity components.

spectra suggest that at least the so-called spherical outflow is traced by $\mathrm{CN}$.

In Figure 3, spectra of the $J=3 \rightarrow 2$ and $5 \rightarrow 4$ transitions of CS are displayed. The CS profiles both have very broad linewidths $\left(\Delta \mathrm{V}_{1 / 2} \sim 70 \mathrm{~km} \mathrm{~s}^{-1}\right)$, indicating the presence of all three velocity components. The $J=5 \rightarrow 4$ line in particular has the boxlike shape, resembling the $J=2 \rightarrow 1$ transition of CO (see Figure 1), with red and blue outflows less obvious because of the reduced signal-to-noise ratio.

In Figure 4, the spectra of $\mathrm{HCO}^{+}$are presented. The $J=1 \rightarrow 0$ and $J=2 \rightarrow 1$ transitions (top two panels) have broad line profiles $\left(\Delta V_{1 / 2} \sim 70 \mathrm{~km} \mathrm{~s}^{-1}\right)$, indicative of all three outflows in VY CMa. (The $J=2 \rightarrow 1$ line near $178 \mathrm{GHz}$ has a poorer signal-to-noise ratio because it lies close to the $183 \mathrm{GHz}$ water line.) The other two transitions are contaminated by the $J_{\mathrm{Ka}, \mathrm{Kc}}=$ $13_{3,11}-13_{2,12}$ and $10_{4,6}-10_{3,7}$ lines of $\mathrm{SO}_{2}$ near 267537 and $356755 \mathrm{MHz}$, respectively. The line profiles are too broad $\left(\Delta V_{1 / 2} \sim 100 \mathrm{~km} \mathrm{~s}^{-1}\right)$ to arise from a single transition, and therefore must be blends of features. A representative $\mathrm{SO}_{2}$ line profile, observed at $244254 \mathrm{MHz}$ and scaled in velocity and

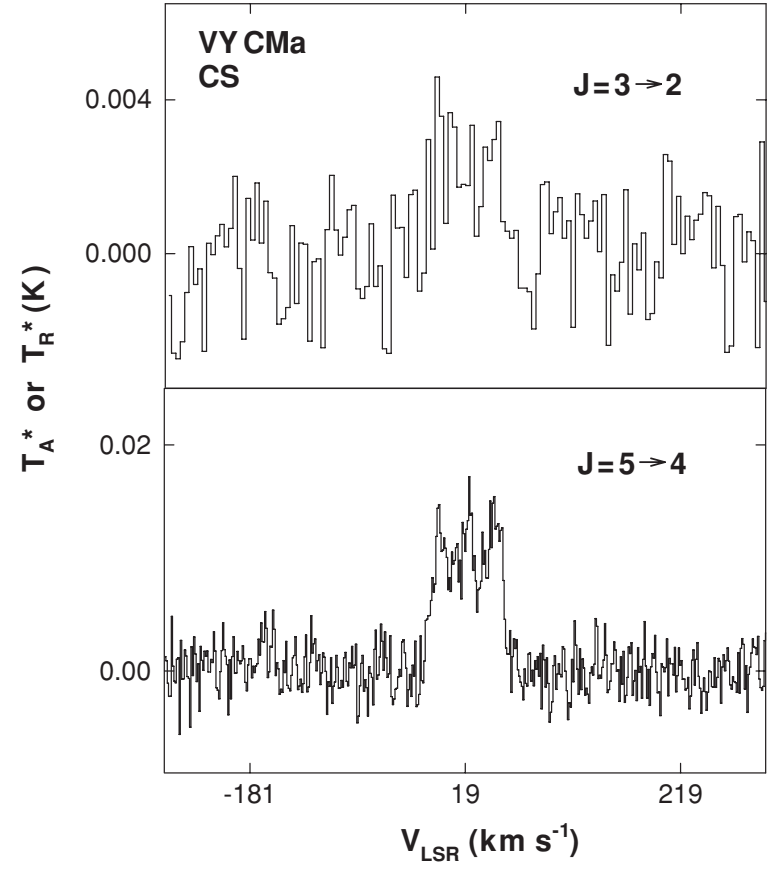

Figure 3. Spectra of the $J=3 \rightarrow 2$ and $5 \rightarrow 4$ transitions of CS, observed with the ARO $12 \mathrm{~m}$ at $147 \mathrm{GHz}\left(T\right.$ scale: $\left.T_{R}{ }^{*}\right)$ and the ARO SMT at $245 \mathrm{GHz}(T$ scale: $T_{A}^{*}$ ), respectively, toward VY CMa. Spectral resolution is $1 \mathrm{MHz}$ for the $J=5 \rightarrow 4$ line and $2 \mathrm{MHz}$ for the $J=3 \rightarrow 2$ transition. These spectra, which confirm the presence of this molecule in this circumstellar shell, have broad linewidths $\left(\Delta V_{1 / 2} \sim 70 \mathrm{~km} \mathrm{~s}^{-1}\right)$. The $J=5 \rightarrow 4$ line in particular has a boxlike shape with blue and redshifted "horns" as well as a central feature, indicating the presence of three distinct velocity components.

brightness temperature to match the contaminating emission, is superimposed over the spectra in gray to illustrate the blending. Additional line intensity from other than $\mathrm{SO}_{2}$ is clearly visible. Note that $\mathrm{SO}_{2}$ has a distinctive line shape similar to that of $\mathrm{HNC}$ (see Figure 2).

For comparison, $\mathrm{CO}$ and $\mathrm{HCN}$ spectra were measured toward other O-rich circumstellar shells: the supergiant NML Cyg and the AGB stars W Hya, TX Cam, and IK Tau. The $J=3 \rightarrow 2$ and $J=1 \rightarrow 0$ transitions of $\mathrm{HCN}$ in these objects are displayed in Figure 5 . The $J=1 \rightarrow 0,2 \rightarrow 1$, and $6 \rightarrow 5$ lines of $\mathrm{CO}$ were observed, as well, with the exception of the $J=6 \rightarrow 5$ line in IK Tau. Representative spectra are shown in Figure 6 for TX Cam and W Hya. The $J=6 \rightarrow 5$ transition of CO had not been previously observed in either of these objects. As shown in both figures, the line profiles for $\mathrm{HCN}$ and $\mathrm{CO}$ arising in these circumstellar shells are fairly symmetric and suggest one stellar wind component. (The structure in the $J=1 \rightarrow 0$ and $2 \rightarrow 1$ lines of CO in TX Cam arises from Galactic contamination.) Line parameters for $\mathrm{CO}$ and $\mathrm{HCN}$ in these sources are listed in Table 2.

\section{ANALYSIS}

In order to determine molecular abundances and distributions, emission from the observed species was modeled using the nonLTE radiative transfer code of Bieging \& Tafalla (1993). This code utilizes the Sobolev approximation to solve the statistical equilibrium equations of molecular energy levels in discrete shells of a spherically symmetric circumstellar envelope. The molecular distribution is then convolved with the given telescope beam at the distance of the object.

Table 3 lists the stellar parameters used for modeling each star. A common $\mathrm{H}_{2}$ density profile was employed (based on 


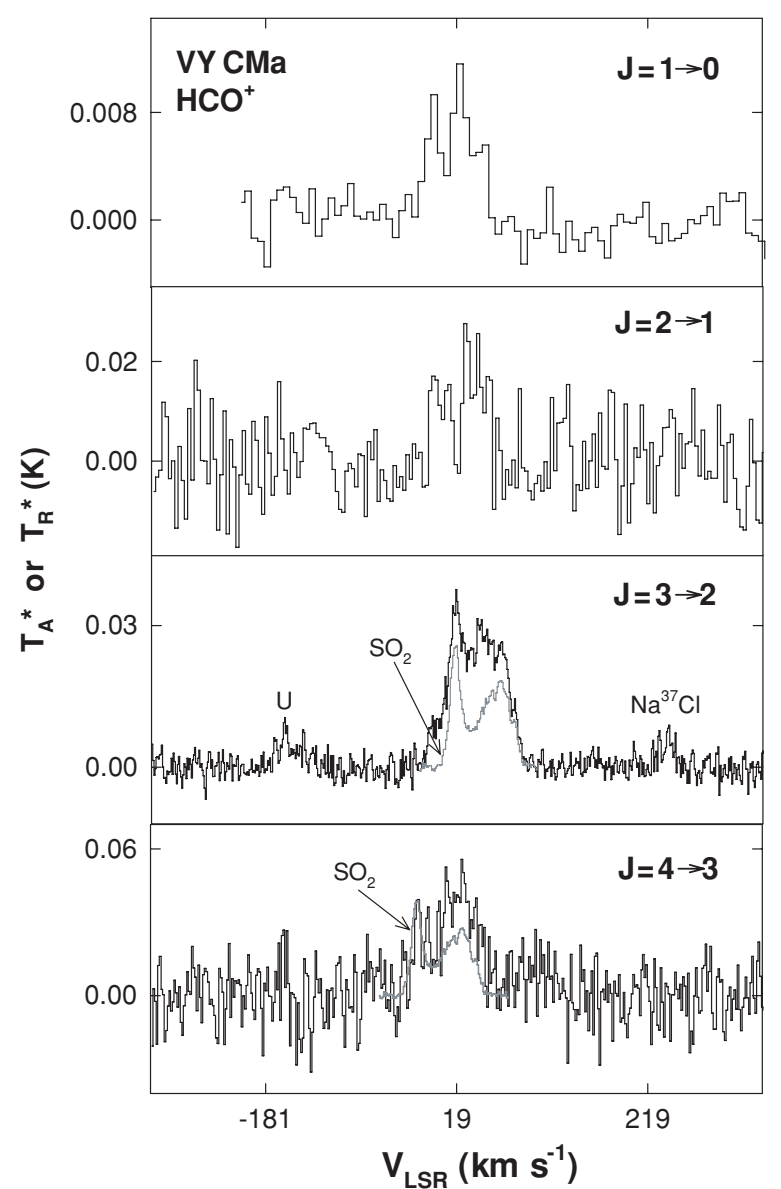

Figure 4. Spectra of the $J=1 \rightarrow 0,2 \rightarrow 1,3 \rightarrow 2$ and $4 \rightarrow 3$ transitions of $\mathrm{HCO}^{+}$observed toward VY CMa at $89 \mathrm{GHz}, 178 \mathrm{GHz}, 267 \mathrm{GHz}$, and 356 $\mathrm{GHz}$, using the ARO $12 \mathrm{~m}$ (2 and $3 \mathrm{~mm}$ lines in units of $T_{R}^{*}$; spectral resolution $2 \mathrm{MHz}$ ) and the ARO SMT ( 1 and $0.8 \mathrm{~mm}$ lines in units of $T_{A}^{*}$; spectral resolution $1 \mathrm{MHz}$ and $2 \mathrm{MHz}$ (smoothed), respectively). The $3 \rightarrow 2$ and $4 \rightarrow 3$ lines are contaminated by transitions of $\mathrm{SO}_{2}$, which broaden and distort the line profiles. A representative profile of $\mathrm{SO}_{2}$, taken from previous observations by Ziurys et al. (2007), is overlaid on the data in gray and can account for some, but not all, of the observed emission. Features due to $\mathrm{Na}^{37} \mathrm{Cl}$ and an unidentified molecule, labeled "U," are present in the $J=3 \rightarrow 2$ spectrum.

Kemper et al. 2003):

$$
\rho(r)=\frac{\dot{M}}{4 \pi v_{\exp }\left(1-\frac{R_{*}}{r}\right) r^{2}},
$$

where $\dot{M}$ is the $\mathrm{H}_{2}$ mass-loss rate, $v_{\text {exp }}$ is the terminal expansion velocity, and $R_{*}$ is the stellar radius. The calculation is initiated at $r_{\text {inner }}$, and rotational levels $(J \leqslant 30)$ in the ground and first vibrational states are considered. Energy levels and Einstein A coefficients are derived from spectroscopic constants and dipole moments reported in the literature, and a black-body radiation field due to dust is also included ( $T_{\text {dust }}$ at $\left.r_{\text {inner }}\right)$. Collisional excitation rates are those tabulated by Schöier et al. (2005) from the following publications: CO: Flower (2001); HCN and HNC: Green \& Thaddeus (1974); CS: Turner et al. (1992); $\mathrm{HCO}^{+}$: Flower (1999). For CN, the HCN rates were employed after correcting for the fine and hyperfine structure.

The gas kinetic temperature profiles, presented in Table 3, are inferred from past studies, which calculated the profiles self-consistently, accounting for both collisional and radiative heating and cooling. Since no such calculations have been published for IK Tau and TX Cam, we used the profile of GX
Mon, an oxygen-rich AGB star of comparable size, mass-loss rate, and stellar temperature (Justtanont et al. 1994).

Molecular distributions are described by the Gaussian function

$$
f(r)=f_{0} e^{-\left(r / r_{\text {outer }}\right)^{2}},
$$

where $f_{0}$ is the abundance at $r_{\text {inner }}$, and $r_{\text {outer }}$ is the radius where the abundance decreases by a factor of $1 / e$. In the case of $\mathrm{HCO}^{+}$, a shell distribution was also considered, described by the expression

$$
f(r)=f_{0} e^{-\left(\left(r-r_{\text {shell }}\right) / r_{\text {outer }}\right)^{2}} .
$$

In this function, $f_{0}$ indicates the peak abundance, which occurs at $r_{\text {shell }}$, and $r_{\text {outer }}$ is the distance from $r_{\text {shell }}$ where the abundance falls by a factor of $1 / e$.

For individual molecules in each envelope, the value of $r_{\text {outer }}$ was varied in increments of $3^{\prime \prime}$, and the abundance, $f_{0}$, was subsequently adjusted to achieve the best agreement between the observed and the predicted emission, determined by visual inspection. When available, emission from past published studies was also considered in the model fitting (CO: Kemper et al. 2003; Kerschbaum \& Olofsson 1999; Knapp et al. 1998; Olofsson et al. 1991; Teyssier et al. 2006; HCN: Nercessian et al. 1989; Bujarrabal et al. 1994; Lindqvist et al. 1988; Lindqvist et al. 1992; Bieging et al. 2000; CN: Bachiller et al. 1997). The best-fit values of $f_{0}$ and $r_{\text {outer }}$ for each observed species are given in Tables 4-6. Because the abundance does not change significantly until $r=r_{\text {outer }}, f_{0}$ represents the average abundance throughout the shell (i.e., $f_{0}=f$ ). Our analysis suggests that the derived source sizes and abundances have absolute uncertainties of at most $30 \%$, based on the quality of the fits to the line profiles.

In order to avoid circularity in our analysis of $\mathrm{CO}$ fractional abundances, it was necessary to adopt mass-loss rates that were derived from methods other than $\mathrm{CO}$ emission modeling. One commonly used technique for determining stellar mass-loss rates is to assume a $\mathrm{CO}$ abundance and outer radius, and then predict CO rotational emission line intensities as the mass-loss rate is varied. The "best" mass-loss rate is then selected based on agreement between observed and predicted emission lines (see, for example, Schöier et al. 2002). In our analysis, we use massloss rates found from dust radiative transfer studies and $\mathrm{H}_{2} \mathrm{O}$ emission modeling (VY CMa: Humphreys et al. 2005; NML Cyg: van Loon et al. 2005; IK Tau: van Loon et al. 2005; Jura \& Kleinmann 1989; TX Cam: van Loon et al. 2005; Olivier et al. 2001; Schutte \& Tielens 1989; Jura \& Kleinmann 1989; W Hya: Justtanont et al. 2005); it should be noted that these mass-loss rates tend to be in good agreement with $\mathrm{CO}$ emission-derived values.

Molecular line profiles from VY CMa suggest that emission arises from at least three kinematically distinct outflows, as shown in Figures 1-3 and discussed previously. It is possible to determine which component(s) are present in a given spectral profile by inspection of the line shape and velocity shift. Since each outflow region possesses a unique chemistry, we separately modeled emission in the three regions. For the central, spherical outflow, the model parameters given in Table 3 were used. In modeling of the red and blue flows, a spherically symmetric envelope is still assumed in the statistical equilibrium calculation. Instead of convolving the entire spherical envelope with the telescope beams, only conical sections of the envelope are integrated. In our analysis, the redshifted jet is best fitted by a cone directed $45^{\circ}$ off the line-of-sight away from the observer with an opening angle of $45^{\circ}$ and an expansion velocity 


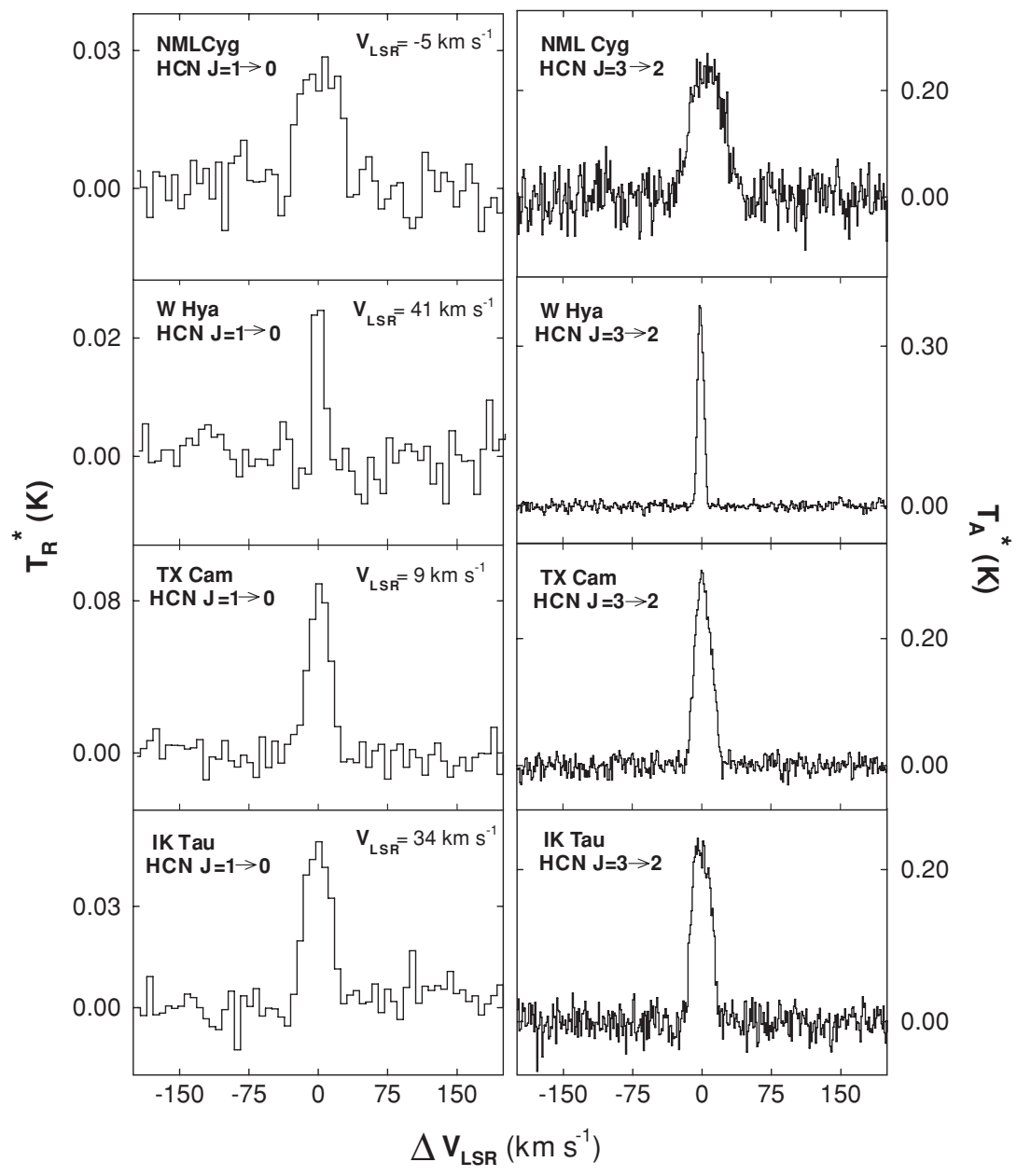

Figure 5. Spectra of the $J=1 \rightarrow 0$ and $3 \rightarrow 2$ transitions of $\mathrm{HCN}$ at $88 \mathrm{GHz}$ and $265 \mathrm{GHz}$ observed toward the oxygen-rich circumstellar shells of supergiant NML Cyg and the AGB stars WHya, TX Cam, and IK Tau. The $J=1 \rightarrow 0$ lines were measured with the $12 \mathrm{~m}$ in units of $T_{R}^{*}$ with $2 \mathrm{MHz}$ resolution and the $J=3 \rightarrow 2$ spectra with the SMT with $1 \mathrm{MHz}$ resolution, where the temperature scale is $T_{A}^{*}$. The $x$-axis scale is in given as $\Delta \mathrm{V}_{\mathrm{LSR}}$ with respect to the LSR velocities shown for each source above the $J=1 \rightarrow 0$ spectra. The line profiles appear to trace a simple spherical flow in these sources.

of $42 \mathrm{~km} \mathrm{~s}^{-1}$. The blueshifted flow is described by a cone pointing $20^{\circ}$ off the line-of-sight toward the observer with an opening angle of $45^{\circ}$, expanding at a velocity of $38 \mathrm{~km} \mathrm{~s}^{-1}$. The projection angles of the directed jets match the infrared features from Humphreys et al. (2005, 2007) quite well. Arcs 1 and 2 appear to be related to the blueshifted wind, and the Curved Nebulous Tail (CNT) or Northwest Arc is likely to be associated with the redshifted flow (see Smith et al. 2001; Ziurys et al. 2007). Atomic spectra taken at positions across the IR emission features roughly agree in velocity with those of the molecular blue and red winds, further supporting this scenario. Additional mapping of molecular emission in VY CMa, such as that done by Muller et al. (2007), would be very helpful in determining the exact kinematic structure of the outflows in this envelope.

\section{DISCUSSION}

\subsection{VY CMa: A Unique Carbon Chemistry?}

VY CMa appears to exhibit the most active carbon chemistry to date of any O-rich circumstellar shell, considering both supergiants and AGB stars. Furthermore, the six carbon-bearing species found in this object are not uniformly distributed, but occupy different outflows with varying abundances and physical extents, as shown in Table 4. CO is still the most abundant molecule in all three regions, but appears to be most prevalent in the redshifted flow, where $f\left(\mathrm{CO} / \mathrm{H}_{2}\right) \sim 2 \times 10^{-4}$. This abundance is typical for M stars (e.g., Ramstedt et al. 2008). The abundance drops by a factor of 3-5 in the other outflows, however. This molecule is also extended in the envelope out to $\sim 1000 R_{*}$. The modeling suggests a radius of $r \sim 5^{\prime \prime}$ for the redshifted flow $\left(\sim 10^{17} \mathrm{~cm}\right), 6^{\prime \prime}$ for the spherical wind $\left(1.3 \times 10^{17}\right.$ $\mathrm{cm})$ and $9^{\prime \prime}$ for the blueshifted outflows $\left(\sim 2 \times 10^{17} \mathrm{~cm}\right)$, for a source distance of $1.5 \mathrm{kpc}$. CO emission from the spherical flow thus encompasses the full extent of the outer halo of infrared emission, while the blueshifted flow appears to extend slightly beyond Arc 1. The redshifted wind in CO appears to also have a similar spatial extent as the CNT or Northwest Arc (e.g., Smith et al. 2001; Humphreys et al. 2005, 2007).

$\mathrm{HCN}$ is not nearly as extended as $\mathrm{CO}$, with calculated source radii in the range $r \sim 2.3^{\prime \prime}-4.5^{\prime \prime}$ or $0.5-1 \times 10^{17}$ $\mathrm{cm}$, corresponding to $250-500 R_{*}$ (see Table 4). It is the second most abundant C-bearing molecule detected in the VY $\mathrm{CMa}$ and is observed in all three kinematic regions, with $f$ $\left(\mathrm{HCN} / \mathrm{H}_{2}\right) \sim 1.2-7.5 \times 10^{-6}-$ about a factor of $10-30$ less abundant than $\mathrm{CO}$. In contrast, HNC was only observed in the blue and redshifted winds, with $f \sim 4-5 \times 10^{-8}$, resulting in a $[\mathrm{HCN}] /[\mathrm{HNC}]$ ratio of $\sim 150-190$. Such a large ratio indicates that these species are located in warm gas (e.g., Ziurys \& Turner 1986). 


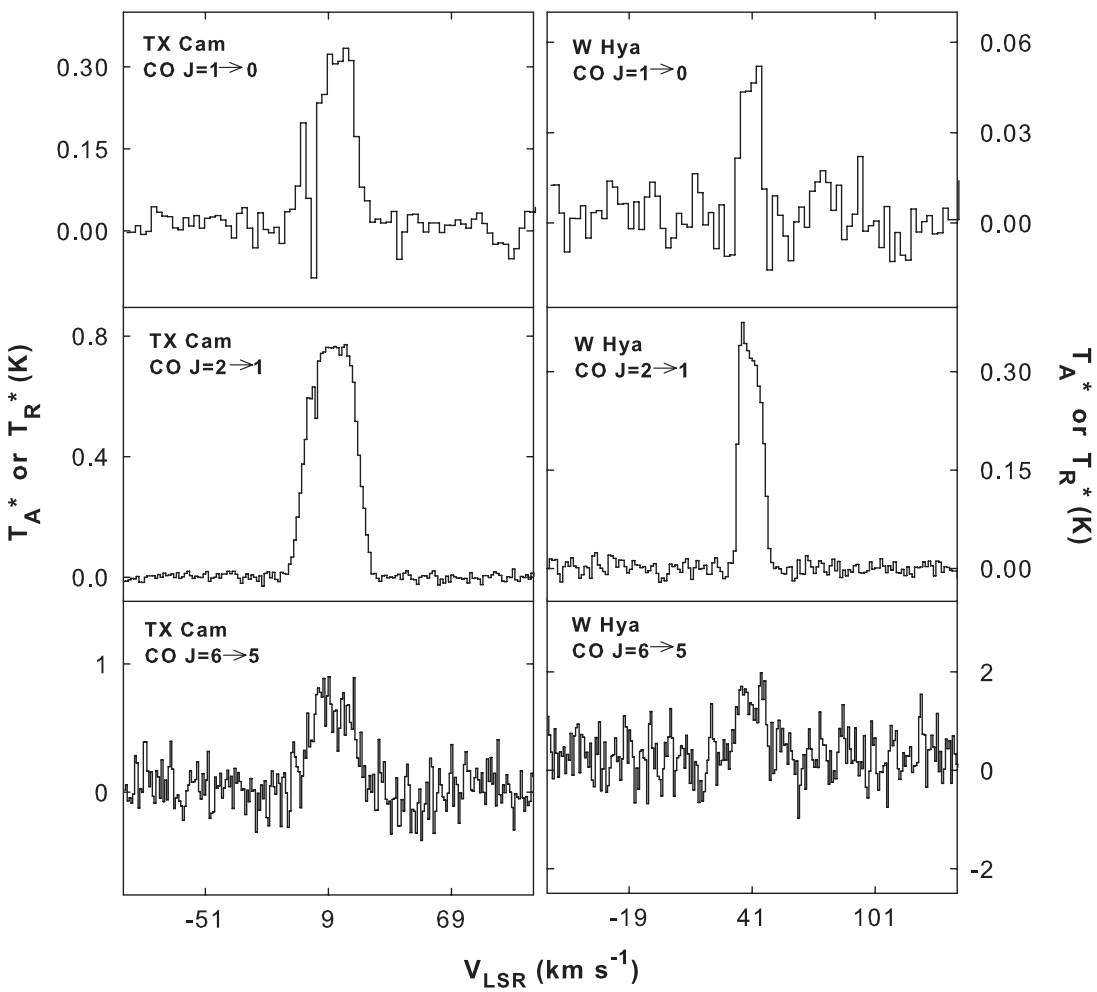

Figure 6. Spectra of the $J=1 \rightarrow 0,2 \rightarrow 1$, and $6 \rightarrow 5$ transitions of CO at $115 \mathrm{GHz}, 230 \mathrm{GHz}$, and $691 \mathrm{GHz}$ measured toward TX Cam and W Hya. The spectra were measured with the SMT in units of $T_{A}^{*}(\mathrm{~J}=2 \rightarrow 1: 1 \mathrm{MHz}$ resolution; $J=6 \rightarrow 5: 1 \mathrm{MHz}$ resolution, smoothed to $2 \mathrm{MHz})$ except for the $J=1 \rightarrow 0$ line, which was observed with the $12 \mathrm{~m} \mathrm{(} T_{R}^{*}$ scale and $1 \mathrm{MHz}$ resolution). There is some Galactic contamination in the $J=1 \rightarrow 0$ and $2 \rightarrow 1$ lines in TX Cam, which appears as sharp "absorption" dips in the data. The $J=6 \rightarrow 5$ transition had not previously observed in these objects, and helps to constrain the CO abundance.

Table 3

Modeling Parameters

\begin{tabular}{lcccccccc}
\hline \hline Source & Distance $(\mathrm{pc})$ & $\dot{M}\left(M_{\odot} \mathrm{yr}^{-1}\right)$ & $V_{\text {exp }}\left(\mathrm{km} \mathrm{s}^{-1}\right)$ & $r_{\text {inner }}(\mathrm{cm})$ & $T_{\text {dust }}(\mathrm{K})$ & $T_{\text {gas }}(\mathrm{K})$ & \multicolumn{1}{c}{} \\
\hline VY CMa & 1500 & $2.0 \times 10^{-4}$ & 20 & $5 \times 10^{15}$ & 400 & $230\left(\frac{10^{16}}{r}\right)^{\gamma}$ & $\mathrm{a}$ & 0.62 \\
NML Cyg & 1700 & $2.0 \times 10^{-4}$ & 31 & $5 \times 10^{15}$ & 400 & $270\left(\frac{10^{16}}{r}\right)^{\gamma}$ & $\mathrm{a}$ & 0.5 \\
TX Cam & 390 & $4.4 \times 10^{-6}$ & 19 & $2 \times 10^{14}$ & 1000 & $220\left(\frac{2 \times 10^{15}}{r}\right)^{\gamma}$ & $\mathrm{b}$ & $1.30\left(r \leqslant 2 \times 10^{15}\right)$ \\
& & & & & & $0.56\left(r \geqslant 2 \times 10^{15}\right)$ \\
IK Tau & 300 & $4.6 \times 10^{-6}$ & 20 & $2 \times 10^{14}$ & 1000 & $220\left(\frac{2 \times 10^{15}}{r}\right)^{\gamma}$ & $\mathrm{b}$ & $1.30\left(r \leqslant 2 \times 10^{15}\right)$ \\
W Hya & 78 & $2.5 \times 10^{-7}$ & 8 & $2 \times 10^{14}$ & 1000 & $425\left(\frac{10^{15}}{r}\right)^{\gamma}$ & $\mathrm{c}$ & $0.56\left(r \geqslant 2 \times 10^{15}\right)$ \\
\hline
\end{tabular}

Notes.

${ }^{\text {a }}$ From Zubko et al. (2004).

${ }^{\mathrm{b}}$ From Justtanont et al. (1994) for GX Mon.

${ }^{\mathrm{c}}$ From Justtanont et al. (2005).

Table 4

Carbon-Bearing Molecules in VY CMa

\begin{tabular}{lccccccc}
\hline \hline Molecule & \multicolumn{3}{c}{ Abundance Relative to $\mathrm{H}_{2}$} & & \multicolumn{3}{c}{ Source Radius ${ }^{\mathrm{a}}$} \\
\cline { 2 - 3 } \cline { 6 - 8 } & Spherical Flow & Red Flow & Blue Flow & & Spherical Flow & Red Flow & Blue Flow \\
\hline $\mathrm{CO}$ & $4.0 \times 10^{-5}$ & $2.0 \times 10^{-4}$ & $8.0 \times 10^{-5}$ & & $1.3 \times 10^{17}\left(6^{\prime \prime}\right)$ & $1.0 \times 10^{17}\left(4.5^{\prime \prime}\right)$ & $1.9 \times 10^{17}\left(9^{\prime \prime}\right)$ \\
$\mathrm{HCN}$ & $1.2 \times 10^{-6}$ & $7.5 \times 10^{-6}$ & $7.5 \times 10^{-6}$ & & $1.0 \times 10^{17}\left(4.5^{\prime \prime}\right)$ & $5.0 \times 10^{16}\left(2.3^{\prime \prime}\right)$ & $5.5 \times 10^{16}\left(2.5^{\prime \prime}\right)$ \\
$\mathrm{HNC}$ & & $5.0 \times 10^{-8}$ & $4.0 \times 10^{-8}$ & & & $3.0 \times 10^{16}\left(1.4^{\prime \prime}\right)$ & $3.0 \times 10^{16}\left(1.4^{\prime \prime}\right)$ \\
$\mathrm{CS}$ & $2.0 \times 10^{-7}$ & $6.0 \times 10^{-7}$ & $4.5 \times 10^{-7}$ & & $1.0 \times 10^{16}\left(0.4^{\prime \prime}\right)$ & $1.2 \times 10^{16}\left(0.5^{\prime \prime}\right)$ & $1.4 \times 10^{16}\left(0.6^{\prime \prime}\right)$ \\
$\mathrm{HCO}^{+}$ & $4.0 \times 10^{-9}$ & $1.6 \times 10^{-8}$ & $1.0 \times 10^{-8}$ & & $2.0 \times 10^{17}\left(9^{\prime \prime}\right)^{\mathrm{b}}$ & $1.9 \times 10^{17}\left(9^{\prime \prime}\right)$ & $2.3 \times 10^{17}\left(10^{\prime \prime}\right)$ \\
$\mathrm{CN}$ & $1.8 \times 10^{-8}$ & & & & $1.2 \times 10^{17}\left(5.5^{\prime \prime}\right)$ & & \\
\hline
\end{tabular}

Notes.

a Source radius is $r_{\text {outer }}$ in units of $\mathrm{cm}(")$.

b Alternative model is a shell distribution with peak abundance of $f_{0}=5 \times 10^{-9}$ at $r_{\text {shell }}=1.4 \times 10^{17} \mathrm{~cm}\left(6.3^{\prime \prime}\right)$ with $r_{\text {outer }}=8.0 \times 10^{16} \mathrm{~cm}\left(3.5^{\prime \prime}\right)$, see text. 
The presence of extensive hyperfine structure in the spectra of $\mathrm{CN}$, along with the low brightness temperatures, makes discernment of individual velocity components very uncertain for VY CMa. The CN data were consequently fitted only with a spherical component, also folding in the $30 \mathrm{~m}$ spectrum of the $N=2 \rightarrow 1$ transition from Bachiller et al. (1997). The resulting abundance is $f \sim 1.8 \times 10^{-8}$ for a source radius of $r \sim 5.5^{\prime \prime}$ or $600 R_{*}$. This radical thus appears to be slightly more extended than $\mathrm{HCN}$, indicating that $\mathrm{CN}$ is a photodestruction product of $\mathrm{HCN}$.

In contrast to $\mathrm{CO}$ and $\mathrm{HCN}$, our analysis suggests that the spatial distribution of CS is relatively confined with $r \sim 0.5^{\prime \prime}$, or $60 R_{*}$, although it is present in all three winds (see Table 4). Its abundance falls in the range $f \sim 2-6 \times 10^{-7}$, making it the third most abundant carbon-bearing molecule observed in VY $\mathrm{CMa}$. This species is likely to be created in photospheric shocks and then processed into other chemical species in the expanding envelope.

$\mathrm{HCO}^{+}$appears to be present in all three outflows, with a spatial extent at least comparable to that of $\mathrm{CO}\left(r \sim 1000 R_{*}\right.$; see Table 4). The modeling suggests an abundance of $1-2 \times 10^{-8}$ in the red- and blueshifted winds. The spherical wind has a somewhat lower $\mathrm{HCO}^{+}$abundance of $f \sim 4 \times 10^{-9}$-but with a radius of $9^{\prime \prime}\left(2 \times 10^{17} \mathrm{~cm}\right)$. This component can also be reproduced by a shell model with $r_{\text {shell }} \sim 6.3^{\prime \prime}$ and a comparable abundance (see Table 4). The coincident spatial distributions of $\mathrm{CO}$ and $\mathrm{HCO}^{+}$point to a photochemical origin of $\mathrm{HCO}^{+}$linked to $\mathrm{CO}$.

Overall, the abundances of the C-bearing molecules appear to be slightly higher in the red- and blueshifted flows than the spherical component. The enhancement in $\mathrm{HCN}$ is the most noticeable - a factor of 6 , while the enrichments in $\mathrm{HCO}^{+}$ and CS are factors of 2-4. The effects of additional shocks within these flows, perhaps caused by these winds impinging on the spherical, more quiescent component, may be causing an increase in molecular abundances. Additional observations are needed to see if this effect is real.

\subsection{Comparison with Other O-Rich Envelopes}

$\mathrm{CO}$ and HCN abundances and source sizes for the AGB stars TX Cam, IK Tau, and W Hya, as well as the O-rich supergiant NML Cyg, are listed in Table 5, along with those of VY CMa for the spherical component. As this table illustrates, the $\mathrm{CO}$ abundance is in general higher for the AGB stars $(f \sim 3-5 \times$ $10^{-4}$ ), relative to the two supergiants by about an order of magnitude. As shown in Table 3, VY CMa and NML Cyg have mass-loss rates of $\dot{M} \sim 2 \times 10^{-4} M_{\odot} \mathrm{yr}^{-1}$-at least a factor of 40 greater than those of the three AGB stars. (These rates have been derived from dust measurements, not $\mathrm{CO}$ emission, as previously discussed.) Although the statistics involve a small sample, these results suggest that the abundance of CO may vary among O-rich circumstellar shells. The spatial extent of the $\mathrm{CO}$ emission also fluctuates. The source radius in CO for TX Cam, at a distance of $390 \mathrm{pc}$ (see Table 3), is comparable to that of the two supergiants-roughly $3 \times 10^{17} \mathrm{~cm}$. The extent of CO in IK Tau is only marginally less $\left(9 \times 10^{16} \mathrm{~cm}\right)$, but that of W Hya is over an order of magnitude smaller $\left(r \sim 8 \times 10^{15} \mathrm{~cm}\right)$. There are of course uncertainties in stellar distances and mass-loss rates, as well as in the model predictions, although other studies have suggested that mass-loss rates are reliable to within factors of \pm 3 (Ramstedt et al. 2008).

The abundances of HCN in this sample of O-rich stars fall in the range $0.9-9 \times 10^{-6}$, relative to $\mathrm{H}_{2}$. In all cases the source size in $\mathrm{CO}$ is larger than that of HCN by roughly 1.4-3 (Table 5). There is no obvious difference in HCN abundances between the supergiants and AGB stars; the values in VY CMa and NML Cyg lie midrange of those in the AGB stars. The HCN abundance does not appear within this sample to increase with mass-loss rate (see Olofsson et al. 1991).

Another comparison is the $[\mathrm{HCN}] /[\mathrm{CO}]$ ratio, which is also given in Table 5. Values fall in the range $[\mathrm{HCN} / \mathrm{CO}] \sim 0.03-$ 0.01 , except for $\mathrm{W}$ Hya, where it is lower by about a factor of 10 from others (0.003). The ratios in the directed outflows are also somewhat higher; $[\mathrm{HCN}] /[\mathrm{CO}]$ is 0.09 in the blueshifted flow, for example.

Some differences in abundances between the supergiants and the AGB stars are perhaps expected. VY CMa and supergiants in general appear to have "macroturbulent" velocity structures probably caused by supermassive convection cells (Humphreys et al. 2007; Lim et al. 1998). The likelihood of the effects of shocks should be considerably higher in supergiants relative to AGB stars. In fact, the chemical composition of VY CMa most closely resembles that of certain oxygen-rich protoplanetary nebulae such as OH 231.8+4.2 and IRAS 19312+1950 (e.g., Omont et al. 1993; Bujarrabal et al. 1994; Sánchez-Contreras et al. 2004; Nakashima \& Deguchi 2005). Although these objects follow completely different evolutionary tracts, they all contain high velocity outflows with evidence for shocked material.

It should also be noted that line shapes for these objects may be deceptive, as has been found in the case of VY CMa. A more complex structure could be hidden in the profiles of NML Cyg, for example, which might be more apparent in other molecular transitions. The CO line profiles in TX Cam are anomalous, as well. Although the appearance of the profiles suggest optically thick spectra in TX Cam (see Figure 5), our analysis and also that

Table 5

$\mathrm{CO}$ and $\mathrm{HCN}$ Abundances and Distributions in O-Rich Stars

\begin{tabular}{|c|c|c|c|c|c|c|c|}
\hline \multirow[t]{2}{*}{ Source } & \multicolumn{3}{|c|}{$\mathrm{CO}$} & \multicolumn{3}{|c|}{$\mathrm{HCN}$} & \multirow[t]{2}{*}{$\mathrm{f}[\mathrm{HCN} / \mathrm{CO}]$} \\
\hline & $f\left[\mathrm{CO} / \mathrm{H}_{2}\right]$ & $r_{\text {outer }}(\mathrm{cm})$ & $\theta_{\mathrm{s}}\left({ }^{\prime \prime}\right)$ & $f\left[\mathrm{HCN} / \mathrm{H}_{2}\right]$ & $r_{\text {outer }}(\mathrm{cm})$ & $\theta_{\mathrm{s}}\left({ }^{\prime \prime}\right)$ & \\
\hline VY CMa & $4 \times 10^{-5 \mathrm{a}}$ & $1.3 \times 10^{17 \mathrm{a}}$ & $12^{\mathrm{a}}$ & $1.2 \times 10^{-6 \mathrm{a}}$ & $1.0 \times 10^{17 \mathrm{a}}$ & $9^{\mathrm{a}}$ & $0.03^{\mathrm{a}}, 0.04^{\mathrm{b}}, 0.09^{\mathrm{c}}$ \\
\hline NML Cyg & $8 \times 10^{-5}$ & $3.6 \times 10^{17}$ & 28 & $2.0 \times 10^{-6}$ & $1.5 \times 10^{17}$ & 11 & 0.03 \\
\hline TX Cam & $3.2 \times 10^{-4}$ & $3.0 \times 10^{17}$ & 100 & $9 \times 10^{-6}$ & $2.0 \times 10^{17}$ & 69 & 0.03 \\
\hline IK Tau & $5.0 \times 10^{-4}$ & $9.0 \times 10^{16}$ & 40 & $4 \times 10^{-6}$ & $3.0 \times 10^{16}$ & 13 & 0.01 \\
\hline W Hya & $3.0 \times 10^{-4}$ & $8.0 \times 10^{15}$ & 14 & $9 \times 10^{-7}$ & $3.0 \times 10^{15}$ & 5 & 0.003 \\
\hline
\end{tabular}

Notes.

${ }^{a}$ Spherical flow.

${ }^{b}$ Redshifted outflow.

${ }^{\mathrm{c}}$ Blueshifted outflow. 
of Ramstedt et al. (2008) only produce lines that are optically thin. A more complex kinematic structure in the envelope may be creating a line profile that has a misleading "thick" shape.

\subsection{Non-Equilibrium Shock Chemistry Versus Photodissociation Processes}

A summary of abundances for the six C-containing species studied here is given in Table 6, along with the theoretical predictions of circumstellar chemistry in O-rich stars considering photospheric shocks (Duari et al. 1999; Cherchneff 2006) and photochemical production (Willacy \& Millar 1997; Mamon et al. 1987). Additionally listed are typical abundances for C-rich stars. Given the morphology of supergiants such as VY CMa, a direct comparison between such models and the observations is likely to be overly simplistic. The current shock models, for example, predict abundances at 2-5 $R_{*}$, and these observations encompass the entire envelope. However, these models are the best that are currently available and some relative evaluation is warranted.

As the table summarizes, the shock models can account for the concentrations of $\mathrm{CO}, \mathrm{HCN}$, and $\mathrm{CS}$ in both types of Orich envelopes (Cherchneff 2006; Duari et al. 1999). In these calculations, $\mathrm{CO}$ is produced via a trimolecular reaction $\mathrm{C}+\mathrm{O}$ $+\mathrm{M}$, where $\mathrm{M}$ is a third body. $\mathrm{HCN}$ enhancement is thought to occur via $\mathrm{CN}$, which is created behind the shock front from the reactions $\mathrm{N}+\mathrm{CH}$ and $\mathrm{N}+\mathrm{CS}$. The process $\mathrm{CO}+\mathrm{S}$ leads to $\mathrm{CS}$. The one exception is the case of $\mathrm{CO}$ in the supergiants. The shock models give $f\left(\mathrm{CO} / \mathrm{H}_{2}\right) \sim 5-6 \times 10^{-4}$ in O-rich envelopes (see Table 6) - about an order of magnitude higher than what is observed in VY CMa and NML Cyg. Note that aperture synthesis maps toward W Hya, IK Tau, and TX Cam with $\sim 0.5^{\prime \prime}-3^{\prime \prime}$ resolution also indicate an inner shell, shock origin of HCN (Marvel 2005; Muller et al. 2008).

The photochemical models also can account for the abundance of CS in VY CMa. In this case, however, significant production of this species is predicted in the outer envelope from the dissociative electron recombination of $\mathrm{HCS}^{+}$(Willacy \& Millar 1997). The confined spatial distribution of CS in VY $\mathrm{CMa}$ is evidence against this interpretation. The photochemical predictions are clearly too low in the case of $\mathrm{HCN}$ by at least an order of magnitude. In this scenario, the photodestruction products $\mathrm{CH}$ and $\mathrm{CH}_{3}$ are the source of carbon for $\mathrm{HCN}$. Neutral-neutral reactions of $\mathrm{NO}+\mathrm{CH}$ or $\mathrm{CH}_{3}+\mathrm{N}$ lead to this species.

The shock models successfully produce the $\mathrm{HCN} / \mathrm{CO}$ ratio as well. These calculations predict $[\mathrm{HCN} / \mathrm{CO}] \sim 0.004-0.02$ at $r>2 R_{*}$ (Duari et al. 1999; Cherchneff 2006), in good agreement with most of the observations. The photochemical approach (Willacy \& Millar 1997) estimates [HCN]/[CO] 0.0003-0.0007-considerably lower than the observed values. The slightly higher $[\mathrm{HCN}] /[\mathrm{CO}]$ ratio of 0.09 , observed in the blueshifted wind, may result from the creation of HCN by additional shocks within this flow.

Either type of model can account for the abundance of $\mathrm{CN}$, to within an order of magnitude. However, it is fairly clear that photodissociation of HCN is a major origin of this radical in C-rich circumstellar shells (e.g., Bachiller et al. 1997), and this source may also be viable in O-rich envelopes. The agreement between the spatial distributions of $\mathrm{CN}$ and $\mathrm{HCN}$ in VY CMa suggests such a connection. Willacy \& Millar (1997) also predict additional photochemical pathways to this radical from $\mathrm{CH}_{3} \mathrm{CN}$ and $\mathrm{HCNH}^{+}$in the regions closer to the star, but these production routes may not be necessary.
Photochemical models predict the abundance of $\mathrm{HCO}^{+}$in VY CMa reasonably well. Calculations by Mamon et al. (1987) suggest $f \sim 2 \times 10^{-8}$ for a mass-loss rate of $10^{-4} M_{\odot} \mathrm{yr}^{-1}$, in agreement with the data for VY CMa. They also predict two pathways for $\mathrm{HCO}^{+}$formation: $\mathrm{C}^{+}+\mathrm{H}_{2} \mathrm{O}$ and $\mathrm{H}_{3}{ }^{+}+\mathrm{CO}$. The latter reaction replenishes the amount of $\mathrm{HCO}^{+}$in the outer envelope, which decreases as $\mathrm{H}_{2} \mathrm{O}$ is photodissociated. Observations suggest that this ion is at least as extended as $\mathrm{CO}$, supporting this scheme. Willacy \& Millar (1997) predict a similar peak abundance for this ion $\left(f \sim 5 \times 10^{-8}\right)$ and an extended source at large radial distances $\left(r \sim 10^{18} \mathrm{~cm}\right)$ from the star.

The HNC abundance of $f \sim 4 \times 10^{-8}$ in VY CMa is reproduced by photochemical calculations, but not the [HCN]/ [HNC] ratio of 150-190. Such models predict an efficient production of $\mathrm{HNC}$ via the process $\mathrm{C}^{+}+\mathrm{NH}_{3}$, leading to an $[\mathrm{HCN}] /[\mathrm{HNC}]$ ratio of $\sim 3-19$ (Willacy \& Millar 1997; Nejad \& Millar 1988). This ratio is thought to be an excellent diagnostic for photochemically induced synthesis of carbonbearing species. The distribution of HNC is also confined in VY $\mathrm{CMa}\left(r \leqslant 150 R_{*}\right)$, indicating an origin closer to the photosphere, but there are currently no abundance predictions for this species from shock models.

There have been theoretical calculations predicting that parent molecules such as $\mathrm{CO}, \mathrm{HCN}$, and $\mathrm{CS}$ may have constant circumstellar abundances nearly independent of the $\mathrm{C} / \mathrm{O}$ ratio in AGB stars (Cherchneff 2006). The rationale for this theory is that such species are all formed in shocks near the stellar photosphere. Presumably "freeze-out" locks the abundances throughout the majority of the shell thereafter (e.g., Scalo \& Slavsky 1980). For comparison, representative abundances for carbon-bearing species in $\mathrm{C}$-rich envelopes are also given in Table 6. The abundances for CS, CO, and $\mathrm{HCN}$ in the O-rich AGB stars are at most a factor of 4 lower than in the C-rich case, but are an order of magnitude smaller for the supergiants. The shells of supergiant stars probably represent a separate class. The abundance of $\mathrm{CN}$ is also significantly higher in the $\mathrm{C}$-rich case. Studies of this radical by Bachiller et al. (1997) indicate that $[\mathrm{CN}] /[\mathrm{HCN}]$ is $\sim 0.04$ in O-rich stars versus $\sim 0.45$ in C-rich objects. Our observations of VY CMa suggests a [CN]/ $[\mathrm{HCN}]$ ratio of $\sim 0.01$ in this star, in good agreement with this study.

\section{CONCLUSIONS}

These new observations have increased the inventory of Cbearing molecules to six in the circumstellar envelope of VY $\mathrm{CMa}$, making this object the richest M-type envelope to date for carbon chemistry. Furthermore, the spectra obtained from VY CMa show three distinct outflows with differing molecular abundances, adding another degree of chemical complexity. These results suggest that detailed studies of other O-rich stars might be fruitful and could reveal more than the usual spherical expanding envelope assumed for these sources, as well as new $\mathrm{C}$-bearing species such as $\mathrm{HCO}^{+}$. A combination of shock and photochemical processes is needed to account for the observed abundances in VY CMa and four other O-rich stars, although more computations are clearly needed to differentiate between these competing scenarios. These data also imply that $\mathrm{CO}$ and $\mathrm{HCN}$ abundances vary among O-rich circumstellar shells, despite having similar $\mathrm{C} / \mathrm{O}$ ratios; supergiants and AGB stars may exhibit somewhat different chemistries, likely to be a result of increased outflows and shocked gas in the supergiant case. The molecular composition of O-rich shells is an area that definitely needs further investigation. 
Table 6

Observed Versus Theoretical Abundances of Carbon-Bearing Molecules

\begin{tabular}{lcccccc}
\hline \hline \multirow{2}{*}{ Molecule } & \multicolumn{3}{c}{ Observed } & & \multicolumn{2}{c}{ Theoretical $^{n}$} \\
\cline { 2 - 3 } \cline { 6 - 7 } & O-Rich Supergiant & O-Rich AGB & C-Rich AGB & & O-Rich Photo-Model $^{\mathrm{a}}$ & O-Rich Shock-Model $^{\mathrm{b}}$ \\
\hline $\mathrm{CO}$ & $6 \times 10^{-5 \mathrm{c}}$ & $4 \times 10^{-4}$ & $7 \times 10^{-4 \mathrm{~d}}$ & & $4 \times 10^{-4}$ & $6 \times 10^{-4}, 5 \times 10^{-4}$ \\
$\mathrm{HCN}$ & $2 \times 10^{-6 \mathrm{c}}$ & $5 \times 10^{-6}$ & $2 \times 10^{-5 \mathrm{e}}$ & & $2 \times 10^{-7}$ & $1 \times 10^{-5}, 2 \times 10^{-6}$ \\
$\mathrm{CS}$ & $2 \times 10^{-7 \mathrm{f}}$ & $\sim 1 \times 10^{-6 \mathrm{~g}}$ & $1 \times 10^{-6 \mathrm{e}}$ & & $3 \times 10^{-7}$ & $2 \times 10^{-6}, 3 \times 10^{-7}$ \\
$\mathrm{HNC}$ & $4 \times 10^{-8 \mathrm{~h}}$ & & $1 \times 10^{-7 \mathrm{e}}$ & & $2 \times 10^{-8}$ & $\ldots$ \\
$\mathrm{CN}$ & $2 \times 10^{-8 \mathrm{f}}$ & $9 \times 10^{-8 \mathrm{i}}$ & $5 \times 10^{-6 \mathrm{e}}$ & & $3 \times 10^{-7}$ & $3 \times 10^{-8}, 2 \times 10^{-10}$ \\
$\mathrm{HCO}^{+}$ & $4 \times 10^{-9 \mathrm{f}}$ & & & $\leqslant 2 \times 10^{-10 \mathrm{j}}$ & $5 \times 10^{-8}, 2-7 \times 10^{-8}$ & $\ldots$ \\
\hline
\end{tabular}

Notes.

a From Willacy \& Millar (1997): average of TX Cam and IK Tau; also $\mathrm{HCO}^{+}$prediction from Mamon et al. (1987)

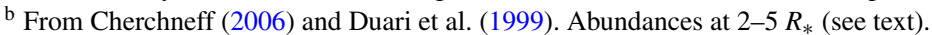

c Average of NML Cyg and spherical flow of VY CMa.

${ }^{\mathrm{d}}$ Ramstedt et al. (2008).

e Olofsson (2005).

${ }^{f}$ Spherical flow of VY CMa.

g Olofsson et al. (1991): abundance in TX Cam.

${ }^{\mathrm{h}}$ Average of red- and blueshifted flows in VY CMa.

${ }^{\mathrm{i}}$ Bachiller et al. (1997): average of TX Cam and IK Tau.

j Agúndez \& Cernicharo (2006): abundance in IRC+10216.

This research is supported by NSF Grant AST-06-07803 and the NASA Astrobiology Institute under cooperative agreement CAN-02-0SS02 issued through the Office of Space Science. E.D.T. thanks the NSF for her graduate fellowship. The authors also thank the staff of the ARO for their assistance, and Eugene Lauria for his efforts in providing the new ARO $0.4 \mathrm{~mm}$ receiver.

\section{REFERENCES}

Agúndez, M., \& Cernicharo, J. 2006, ApJ, 650, 374

Bachiller, R., Fuente, A., Bujarrabal, V., Colomer, F., Loup, C., Omont, A., \& de Jong, T. 1997, A\&A, 319, 235

Bieging, J. H., Shaked, S., \& Gensheimer, P. D. 2000, ApJ, 543, 897

Bieging, J. H., \& Tafalla, M. 1993, AJ, 105, 576

Bujarrabal, V., Fuente, A., \& Omont, A. 1994, A\&A, 285, 247

Cherchneff, I. 2006, A\&A, 456, 1001

Decin, L., Cherchneff, I., Hony, S., Dehaes, S., De Breuck, C., \& Menten, K. M. 2008, A\&A, 480, 431

Duari, D., Cherchneff, I., \& Willacy, K. 1999, A\&A, 341, L47

Flower, D. R. 1999, MNRAS, 305, 651

Flower, D. R. 2001, J. Phys. B: At. Mol. Opt. Phys., 34, 2731

Ford, K. E. S., Neufeld, D. A., Schilke, P., \& Melnick, G. J. 2004, ApJ, 614, 990

Green, S., \& Thaddeus, P. 1974, ApJ, 191, 653

Humphreys, R. M., Davidson, K., Ruch, G., \& Wallerstein, G. 2005, AJ, 129, 492

Humphreys, R. M., Helton, L. A., \& Jones, T. J. 2007, AJ, 133, 2716

Jura, M., \& Kleinmann, S. G. 1989, ApJ, 341, 359

Justtanont, K., Skinner, C. J., \& Tielens, A. G. G. M. 1994, ApJ, 435, 852

Justtanont, K., et al. 2005, A\&A, 439, 627

Kemper, F., Stark, R., Justtanont, K., de Koter, A., Tielens, A. G. G. M., Waters, L. B. F. M., Cami, J., \& Dijkstra, C. 2003, A\&A, 407, 609

Kerschbaum, F., \& Olofsson, H. 1999, A\&AS, 138, 299

Knapp, G. R., Young, K., Lee, E., \& Jorissen, A. 1998, ApJS, 117, 209

Lim, J., Carilli, C. L., White, S. M., Beasley, A. J., \& Marson, R. G. 1998, Nature, 392,575

Lindqvist, M., Nyman, L. Å., Olofsson, H., \& Winnberg, A. 1988, A\&A, 205, L15

Lindqvist, M., Olofsson, H., Winnberg, A., \& Nyman, L. Å 1992, A\&A, 263, 183

Mamon, G. A., Glassgold, A. E., \& Omont, A. 1987, ApJ, 323, 306

Marvel, K. B. 2005, AJ, 130, 261
Melnick, G. J., Neufeld, D. A., Ford, K. E. S., Hollenbach, D. J., \& Ashby, M. L. N. 2001, Nature, 412, 160

Milam, S. N., Apponi, A. J., Woolf, N. J., \& Ziurys, L. M. 2007, ApJ, 668, L131

Milam, S. N., Woolf, N. J., \& Ziurys, L. M. 2009, ApJ, 690, 837

Monnier, J. D., et al. 1997, ApJ, 481, 420

Muller, S., Dinh-V-Trung, , Lim, J., Hirano, N., Muthu, C., \& Kwok, S. 2007, ApJ, 656, 1109

Muller, S., Dinh-V-Trung, , He, J., \& Lim, J. 2008, ApJ, 684, L33

Nakashima, J., \& Deguchi, S. 2005, ApJ, 633, 282

Nejad, L. A. M., \& Millar, T. J. 1988, MNRAS, 230, 79

Nercessian, E., Omont, A., Benayoun, J. J., \& Guilloteau, S. 1989, A\&A, 210, 225

Olivier, E. A., Whitelock, P., \& Marang, F. 2001, MNRAS, 326, 490

Olofsson, H. 2005, in Proc. of the Dusty Molecular Universe: A Prelude to Herschel and ALMA, ed. A. Wilson (Noordwijk: ESA), 223

Olofsson, H., Lindqvist, M., Nyman, L. Å, \& Winnberg, A. 1998, A\&A, 329, 1059

Olofsson, H., Lindqvist, M., Winnberg, A., Nyman, L. Å, \& Nguyen-Q-Rieu, 1991, A\&A, 245, 611

Omont, A., Lucas, R., Morris, M., \& Guilloteau, S. 1993, A\&A, 267, 490

Ramstedt, S., Schöier, F. L., Olofsson, H., \& Lundgren, A. A. 2008, A\&A, 487, 645

Sánchez Contreras, C., Gil de Paz, A., \& Sahai, R. 2004, ApJ, 616, 519

Scalo, J. M., \& Slavsky, D. B. 1980, ApJ, 239, L73

Schöier, F. L., Ryde, N., \& Olofsson, H. 2002, A\&A, 391, 577

Schöier, F. L., van der Tak, F. F. S., van Dishoeck, E. F., \& Black, J. H. 2005, A\&A, 432, 369

Schutte, W. A., \& Tielens, A. G. G. M. 1989, ApJ, 343, 369

Smith, N., Humphreys, R. M., Davidson, K., Gehrz, R. D., Schuster, M. T., \& Krautter, J. 2001, AJ, 121, 1111

Tenenbaum, E. D., Apponi, A. J., Ziurys, L. M., Agúndez, M., Cernicharo, J., Pardo, J. R., \& Guélin, M. 2006, ApJ, 649, L17

Tenenbaum, E. D., Woolf, N. J., \& Ziurys, L. M. 2007, ApJ, 666, L29

Teyssier, D., Hernandez, R., Bujarrabal, V., Yoshida, H., \& Phillips, T. G. 2006, A\&A, 450, 167

Tsuji, T. 1973, A\&A, 23, 411

Turner, B. E., Chan, K., Green, S., \& Lubowich, D. A. 1992, ApJ, 399, 114

van Loon, J. Th., Cioni, M.-R. L., Zijlstra, A. A., \& Loup, C. 2005, A\&A, 438, 273

Willacy, K., \& Millar, T. J. 1997, A\&A, 324, 237

Ziurys, L. M., Milam, S. N., Apponi, A. J., \& Woolf, N. J. 2007, Nature, 447, 1094

Ziurys, L. M., \& Turner, B. E. 1986, ApJ, 302, L31

Zubko, V., Li, D., Lim, T., Feuchtgruber, H., \& Harwit, M. 2004, ApJ, 610, 427 